\title{
Psychosocial correlates of obesity in adolescents: From prevention to intervention
}

\section{Kashmala Qasim}

\section{McMaster University}

In 2005, the Public Health Agency of Canada reported a measured rate of $9.4 \%$ for obesity in youth between the ages of 12 and 17, with the prevalence of adolescents at risk for developing obesity also on the rise. ${ }^{1}$ Adolescent obesity, defined as a body mass index (BMI) at the 99th percentile for the adolescents' age and sex, can be complicated by the co-morbidities of "adult" diseases, including obstructive sleep apnea, hyperlipidemia and type II diabetes leading to a vicious cycle of poor health and persistence of obesity into adulthood. ${ }^{2}$ A recent review suggests that in the developed world the psychosocial effects of adolescent obesity such as mood disorders, low self-esteem and negative body image are highly detrimental. ${ }^{3}$ Thus, in order to combat the population burden of childhood obesity, a more holistic approach is warranted, comprised of lifestyle modification supported by psychosocial-based intervention for overweight adolescents at risk of becoming obese.

The evidence regarding preventative strategies for childhood and adolescent weight gain have been systematically reviewed in the literature, suggesting that increased physical activity, healthy meal planning, reduced television watching as well as parental support, are key features for long-term weight control. ${ }^{4}$ However, less is known regarding the additional role that psychosocial factors can play toward both the development of obesity and its influence on weight loss within already overweight adolescents. For example, an important aspect of managing adolescent obesity is coping with weight-related stereotypes and teasing, which may be internalized through feelings of low self-esteem and depression. Therefore, it is unclear whether such psychosocial vulnerabilities emerge as a result of weight gain or increase the risk of obesity onset. For example, a study by Haines et al. demonstrated that body dissatisfaction and concern with weight were significant predictors of obesity in male and female adolescents. ${ }^{5}$ Specifically, they observed a significant correlation among boys between increased levels of depressive symptoms and prevalence of overweight. ${ }^{5}$ Interestingly, these findings suggest that depression may play a causal role in the development of obesity, and is not exclusively an outcome. ${ }^{6}$

Additionally, Stice and colleagues reported that certain psychological risk factors predicted the emergence of obesity in adolescent girls. ${ }^{6}$ Participants who engaged in harmful weight-loss behaviours such as vomiting or laxative misuse demonstrated a higher risk for obesity onset. ${ }^{6}$ This correlation between weight loss efforts and consequent weight gain might seem counterintuitive. However, researchers suggest that weight-control behaviours may result in elevated metabolic efficiency or even increased binge eating, resulting in weight gain. ${ }^{5}$

Given these findings, prevention programs embedded within a psychosocial framework may be beneficial for adolescents at risk for developing obesity. For example, school-based focus groups and counseling aimed at promoting positive self-image, coping with teasing/bullying and preventing unhealthy weight control behaviors among adolescents of all body types could be initiated. Additionally, adolescents should be encouraged to engage in a variety of physical activities with the notion that exercise enhances endorphin secretion thereby reducing depression levels. ${ }^{7}$

Although $\mathrm{BMI}$ is considered as an imprecise measure of health risk, it may serve as a starting point for classification of obesity onset. ${ }^{8}$ At present, no validated formula exists that can integrate BMI, psychosocial factors and familial history to determine which adolescents are at risk. Therefore, clinicians must utilize evidence-based judgment and evaluate the potential onset of obesity in both normal weight and overweight adolescents by assessing key areas such as parental obesity, sedentary behavior and family mental health history. ${ }^{8}$ 
Ideally, programs should be culturally sensitive and encourage adolescents to express their personal ideals of weight and beauty, which might serve as a social buffer, allowing adolescents to critically appraise media consumption and judge self-worth independent of appearance. ${ }^{9}$ To illustrate, a recent peer-reviewed study found that strength of religious faith among Muslim women was inversely associated with body discontentment, self-objectification, and dietary self-control. ${ }^{9}$ For these women, relationships were mediated by increased use of modest clothing and by reduced media consumption. On a cautionary note, the socio-cultural context of adolescents may only be beneficial if it does not promote attitudes of appearing attractive for the pleasure of others, or cultural practices such as uninterrupted fasting.

Overall, obesity is a multi-factorial illness, influenced by genetic, hormonal and environmental factors. Psychosocial factors alone cannot account for the alarming rates of obesity among adolescents, however, the current obesity epidemic in a fairly constant population illustrates that purely genetic factors are also not the chief culprit and that an integrated approach towards the prevention of pediatric obesity is preferable. ${ }^{10}$

Although treatments such as diet and exercise are effective interventions for managing adolescent obesity, they are a response rather than a solution to a growing health concern. Taking psychosocial variables into consideration may play a mediating role in reducing the rate of overweight adolescents as well as helping those who are overweight to be successful in their weight loss.

\section{Acknowledgments}

I would like to acknowledge the support of my supervisor Dr. Valerie Taylor, as well as my colleague Dr. Irena Milosevic for their constructive review of the manuscript.

\section{References}

1. Hodgson C. Obesity in Canada: A snapshot. Public Health Agency of Canada. Ottawa: Her Majesty the Queen in Right of Canada; 2009.

2. Zeller MH, Modi AC, Moll JG et al. Psychosocial functioning improves following adolescent bariatric surgery. Obesity 2009;17:985-990.

3. Reilly JJ. Obesity in childhood and adolescence: Evidence based clinical and public health perspectives. Postgrad Med J. 2006;82:429-437

4. Fowler $A B$, Kahwati LC. Prevention of treatment of overweight in children and adolescents. Am Fam Physician. 2004;69(11):2591-2599.

5. Haines J, Neumark-Sztainer D, Wall $M$ et al. Personal, behavioural and environmental risk and protective factors for adolescent overweight. Obesity 2007;15:2748-2760.

6. Stice $\mathrm{E}$, Presnell $\mathrm{K}$, Shaw $\mathrm{H}$, et al. Psychological and behavioral risk factors for obesity onset in adolescent girls: A prospective study. J Consult Clin Psychol. 2005;73(2):195-202.

7. Dinas PC, Koutedakis Y, Flouris AD. Effects of exercise and physical activity on depression. Ir J Med Sci. 2011;180:319-325.

8. Barlow SE, Expert Committee. Expert committee recommendations regarding the prevention, assessment and treatment of child and adolescent overweight and obesity: Summary report. Pediatrics 2007;120 Suppl 4:S164-92.

9. Mussap AJ. Strength of faith and body image in Muslim and non-Muslim women. Ment Health Relig Cult. 2009;12(2):121-127.

10. Lobstein T, Baur L, Uauy R et al. Obesity in children and young people: a crisis in public health. Obesity Reviews. 2004;5(1):4-85.

\section{Kashmala Qasim}

Kashmala Qasim is currently pursuing a M.Sc degree in Neuroscience at McMaster University in the McMaster Integrative Neuroscience Discovery and Study program (MINDS). Her area of research focuses on psychological predictors of bariatric surgery outcomes in individuals with obesity. 